\title{
A PARTIAL SURFACE VARIATION FOR EXTREMAL SCHLICHT FUNCTIONS
}

\author{
BY
}

T. L. MCCOY

\begin{abstract}
ABSTRACr. Let a topological sphere be formed from $|z|<1$ by dissecting the circumference into finitely many pairs $\left(I_{j}, I_{j}^{\prime}\right)$ of disjoint arcs, identifying $I_{j}$ and $I_{j}^{\prime}$ in opposite directions and making further identifications among the endpoints. If there exists a meromorphic function $Q(z)$, real and nonnegative on $|z|=1$ and satisfying certain consistency conditions with respect to the dissection (given in detail in our Introduction), then one forms a $Q$-polygon by using the element of length $i d s=\sqrt{Q(z)} d z / z$ to effect the metric identification of the pairs $I_{j}, I_{j}^{\prime}$. In a natural way, $Q$-polygons become Riemann surfaces, thus can be mapped conformally onto the number sphere. When $Q$ is of the form $Q(z)=\sum_{j=-N}^{N} B_{j} z^{j}$, then the corresponding mapping functions, suitably normalized, become the extremal schlicht functions for the coefficient body $V_{N+1}[3$, p. 120].

Suppose that for a given dissection of $|z|=1$ there is a family $Q(z, t)$ of consistent meromorphic functions. For $Q$ sufficiently smooth as a function of $\varepsilon$, we study the variation of the corresponding normalized mapping functions $f(p, \varepsilon)$, using results of [2], and show smoothness of $f$ as a function of $\varepsilon$. Specializing $Q$ to the form above, we deduce the existence of smooth submanifolds of $\partial V_{N+1}$ and obtain a variational formula for the extremal schlicht functions corresponding to motion along these submanifolds.
\end{abstract}

1. Introduction. Notion of a $Q$-polygon. We begin by describing a certain type of topological polygon. Start with the closed unit disc, with the consists of at most one point. Now we identify the arcs of each pair $I_{j}, I_{j}^{\prime}$ in opposite directions, after which we may possibly make additional identifications of endpoints of these arcs. There results a topological surface of finite genus, with the system of identified arcs and endpoints forming the branches and vertices of a tree lying in the surface. We shall suppose from now on that no pair $I_{j}, I_{j}^{\prime}$ separates another pair. This makes the surface a topological sphere.

A $Q$-polygon will be a surface $S$ of the kind described above, but with certain metric conditions determined by a function $Q$. Let us suppose there is a function $Q(z)$, meromorphic in a neighborhood of the unit circumference, and satisfying with regard to the arcs $I_{j}, I_{j}^{\prime}$ the conditions

(i) $Q(z) \geqslant 0$ for $|z|=1$,

Presented to the Society, November 11, 1971 under the title $A$ surface variation for extremal functions; received by the editors November 18, 1975.

AMS (MOS) subject classifications (1970). Primary 30A36.

- American Mathematical Society 1977 
(ii) $Q(z)>0$ except perhaps at endpoints of the arcs,

(iii) $\int_{I_{j}} \sqrt{Q(z)} d z / z=\int_{I_{j}} \sqrt{Q(z)} d z / z$, where we take the positive square root on both sides, and integrate over $I_{j}$ and $I_{j}^{\prime}$ in the counterclockwise sense. Now let $a_{j}$ and $b_{j}$ be the left and right endpoints, respectively, of $I_{j}$ as seen from the origin (that is when we traverse the unit circumference in the counterclockwise sense we encounter $a_{j}$ first) and let $a_{j}^{\prime}, b_{j}^{\prime}$ be the right and left endpoints, respectively, of $I_{j}^{\prime}$ as seen from the origin. We now define what is to be meant by a $Q$-polygon:

Definition 1.1. Let the surface $S$ and the function $Q$ be related as above. Then $S$ is a $Q$-polygon if we identify the interiors of arcs $I_{j}, I_{j}^{\prime}$ according to the metric condition

$$
\int_{a_{j}}^{z} \sqrt{Q(t)} \frac{d t}{t}=-\int_{a_{j}^{\prime}}^{z^{\prime}} \sqrt{Q(t)} \frac{d t}{t}, \quad z \in I_{j}, z^{\prime} \in I_{j}^{\prime},
$$

with the positive square root understood on each side.

Schaeffer and Spencer consider [3, pp. 113ff.] $Q$-polygons where $Q(z)$ is meromorphic on the sphere and vanishes at least once on the unit circumference. They prove that one can use $Q$ to determine a system of local parameters which makes the $Q$-polygon into a Riemann surface (which is therefore a conformal sphere) and it is easy to see that their discussion is equally valid for our slightly more general $Q$-polygons. We describe the local parameters below. First, let us define a function $Z\left(z_{1}, z_{2}\right)$. We set

$$
Z\left(z_{1}, z_{2}\right)=\int_{z_{1}}^{z_{2}} \sqrt{Q(t)} \frac{d t}{t} .
$$

Then:

(a) Let $p_{0}$ be a point lying above $z_{0}$ in the open unit disc. Then a parametric disc about $p_{0}$ will consist of points $p$ lying above a disc $\left|z-z_{0}\right|<\delta$ lying within the open unit disc, and the local parameter will be defined by simple projection on the $z$-plane.

(b) Let $p_{0}$ lie above identified points $z_{0}, z_{0}^{\prime}$ on $I_{j}, I_{j}^{\prime}$, respectively. Then a parametric disc will consist of half-neighborhoods $\Re\left(z_{0}\right), \Re\left(z_{0}^{\prime}\right)$, identified by

$$
Z\left(z_{0}, z\right)=-Z\left(z_{0}^{\prime}, z^{\prime}\right), \quad z \in \Re\left(z_{0}\right), z^{\prime} \in \Re\left(z_{0}^{\prime}\right),
$$

the branches chosen by requiring that $\sqrt{Q}$ be positive on the circumference. We suppose $\Re\left(z_{0}\right), \Re\left(z_{0}^{\prime}\right)$ so small that they lie in the region where $Q$ is analytic and zero-free and do not include any endpoints of arcs. The local parameter will be $\tau(p)$ :

$$
\tau(p)= \begin{cases}Z\left(z_{0}, z\right), & z \in \mathscr{T}\left(z_{0}\right), \\ -Z\left(z_{0}^{\prime}, z^{\prime}\right), & z^{\prime} \in \Re\left(z_{0}^{\prime}\right) .\end{cases}
$$


(c) Let $p_{0}$ lie above identified endpoints $z_{1}, \ldots, z_{v}$. Then a parametric disc about $p_{0}$ will be the union of half-neighborhoods $\Re\left(z_{k}\right)$ of the $z_{k}$, and for the local parameter we take

$$
\tau(p)=\left(Z\left(z_{k}, z\right)\right)^{z / c}, \quad z \in \Re_{k},
$$

for a certain choice of branches. Here,

$$
c=\sum_{k=1}^{\nu}\left(1+c_{k}\right),
$$

$c_{k}$ the order of the zero of $Q$ at $z_{k}$.

Corresponding to each $Q$-polygon, there is a unique function $f(p, v)$, mapping the $Q$-polygon conformally onto the complex number sphere $e$, having its pole at a fixed vertex $v$ of the tree, and having the expansion $f(p, v)=z+a_{2} z^{2}+\ldots$ in terms of a local parameter about the point lying above the origin. In the case considered by Schaeffer and Spencer, such functions which, in addition, have their pole on the unit circumference are precisely the extremal schlicht functions; that is, if

$$
Q(z)=\sum_{k=-N}^{N} B_{k} z^{k}
$$

then the corresponding mapping function has the property that the point $\left(a_{2}, \ldots, a_{N+1}\right)$ lies on the boundary of the coefficient region $\mathfrak{V}_{N+1}$, if it is regular in $|z|<1[3$, p. 120]. For this reason, it is of interest to study the variation of $f$ as we pass from a $Q$-polygon to a neighboring $Q$-polygon. This would lead to a local description of curves lying in the boundary of $\mathfrak{T}_{N+1}$. This program is undoubtedly extremely difficult to carry out in general, and we must content outselves in this paper with obtaining a variational formula for $f$ under very special variations of the $Q$-polygon. Our main results are summarized in Theorem 4.1 below, and in the more specific concluding remarks of our $\$ 6$.

It is clear that the correspondence between $Q$-polygons and functions $Q$ is not one-to-one. Given $Q$, there will in general be a number of topologically different trees which consist of arcs identified according to (1.1). Conversely, there may be many different metric functions $Q$ for a given metric topological polygon.

It is interesting to notice (and routine to verify) that if $Q$ is meromorphic on the sphere, the $Q$-polygon possesses a quadratic differential with representations

$$
\left(Q(\tau) / \tau^{2}\right) d \tau^{2}, \quad d \tau^{2}, \quad\left(c^{2} / 4\right) \tau^{c-2} d \tau^{2}
$$

in terms of local parameters about points lying, respectively, above the open unit disc, interiors of identified arcs, and identified endpoints. 
2. Variation of $Q$-polygons by re-identification of edges of branches. As we have seen in the preceding section, a $Q$-polygon is a Riemann surface, which we denote by $R(Q, T)$, where $T$ is the abstract system of identified arcs and endpoints. Now consider varying $\Re(Q, T)$ by holding all arcs $I_{i}, I_{j}^{\prime}$ fixed, making the same identifications, and letting $Q$ vary smoothly with a parameter $\varepsilon$, in such a way that the metric $Q(z, \varepsilon)$ is still consistent with $T$. We take $Q(z, 0)=Q(z)$, and $\varepsilon$ varying throughout some interval $|\varepsilon| \leqslant \varepsilon_{0}$.

Geometrically, we pass from $R(Q, T)$ to $R(\tilde{Q}, T)(\tilde{Q}(z)=Q(z, \varepsilon))$ by cutting along each branch of the tree, denoted by $\Gamma$, in $R(Q, T)$, then sewing the edges of each cut back together according to the new identification metric $\tilde{Q}$. As seen from $R(Q, T)$ the edges are not quite correctly sewn back together, so that $\Re(\tilde{Q}, T)$ is a slightly distorted copy of $\Re(Q, T)$. We regard each branch $\Gamma_{j}$ of $\Gamma$ as having a left edge, $\Gamma_{j}^{-}$, lying over $I_{j}$, and a right edge $\Gamma_{j}^{+}$lying over $I_{j}^{\prime}$, and think of $\Gamma_{j}^{+}$as being slightly distorted when it is sewn back onto $\Gamma_{j}^{-}$, so that a point $p$ on $\Gamma_{j}^{-}$is re-identified with a point $p+\varepsilon \chi_{j}(p, \varepsilon)$ on $\Gamma_{j}^{+}$.

We have to consider when such a sewing can be realized in terms of conformal mappings from a surface onto itself. The discussion of sewing along a single arc in the number sphere is discussed in [1, pp. 69ff.] and the reader is asked to consult this work for background. The technique is to form a new Riemann surface having a local parameter formed from re-identified half-neighborhoods of $p, p+\varepsilon \chi_{j}(p, \varepsilon)$ on the interiors of arcs. We need to be able to describe new uniformizers about the endpoints of the arcs which will preserve the conformal character of the surface. In the case considered by Courant, it suffices to impose a regularity condition on $\chi$ at the endpoints. In the case where we simultaneously sew along several arcs, it is clear that the $\chi_{j}$ cannot be chosen independently of each other. However, if the sewing map exists, which it does in passage from $\Re(Q, T)$ to $\Re(\tilde{Q}, T)$ [2], it is possible to study the first variation of the mapping function. This is the content of [2], and we summarize the results of that paper below.

Suppose $\mathscr{N}$ is a closed Riemann surface of genus zero, and $\Gamma$ is a tree on

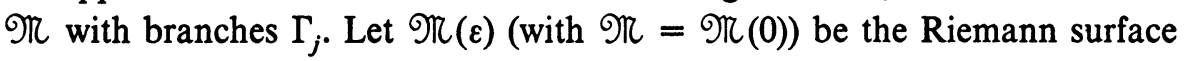
obtained by re-identifying opposite edges of each $\Gamma_{j}$ by the rule that $p \rightarrow p+$ $\varepsilon \chi_{j}(p, \varepsilon)$ as described above. We assume $\Re(\varepsilon)$ is also conformally equivalent to $\mathcal{C}$. Then there exists, for any fixed $p_{0}$ not on $\Gamma$, a unique function $F(p, \varepsilon$; $p_{0}$ ) mapping $\Re(\varepsilon)$ conformally onto $C$ and having at any fixed $p_{0}$ a simple pole with residue 1 and zero translation. If $v$ is a fixed vertex of $\Gamma$, there exists a unique function $f(p, \varepsilon ; v)$ mapping $\Re(\varepsilon)$ conformally onto $\mathcal{C}$, with its pole at $v$ and a normalized expansion

$$
f(p, \varepsilon ; v)=\tau+a_{2} \tau^{2}+\ldots
$$


in terms of a local parameter about $p_{0}$. Indeed,

$$
f(p, \varepsilon ; v)=\frac{1}{F\left(p, \varepsilon ; p_{0}\right)-F\left(v, \varepsilon ; p_{0}\right)}
$$

It will simplify the exposition if we introduce the notion of a regular sewing process.

DEFINITION 2.1. We shall say the sewing process discussed above is regular if:

(i) Each branch $\Gamma_{j}$ is analytic in its interior and Hölder-continuous in its closure, in terms of local parameters.

(ii) There is a parametric patch $D_{j}$ containing the interior of $\Gamma_{j}$ and meeting the endpoints of $\Gamma_{j}$ at a positive angle, in terms of local parameters.

(iii) Let the re-identification $p \rightarrow p+\varepsilon \chi_{j}(p, \varepsilon)$ be described in terms of local parameters by $\tau \rightarrow \tau+\varepsilon r_{j}(\tau, \varepsilon)$. Then $r_{j}(\tau, \varepsilon)$ is analytic in $\tau$ and continuous in $\varepsilon$ for $|\varepsilon| \leqslant \varepsilon_{0}$ and $\tau$ in $\mathscr{D}_{j}$. In addition, $r_{j}(\tau, \varepsilon)$ is bounded independently of $\varepsilon$ on the closure of $\Gamma_{j}$.

THEOREM 2.1. Let $\mathfrak{N}(\varepsilon)$ be derived from $\mathfrak{T}$ by a regular sewing process. In addition, suppose $r_{j}(\tau, \varepsilon)$ satisfies at the endpoints of $\Gamma_{j}$ a Hölder-condition

$$
\left|r_{j}(\tau, \varepsilon)\right|<\left\{\begin{array}{l}
A\left|\tau-\tau\left(a_{j}\right)\right|^{\mu}, \\
A\left|\tau-\tau\left(b_{j}\right)\right|^{\mu},
\end{array} \quad \mu>0\right.
$$

with $A$ independent of $\varepsilon$ as $p$ tends to an endpoint of $\Gamma_{j}$ from within $D_{j}$.

Then, putting

$$
f(p, 0 ; v)=f(p ; v), \quad F\left(p, 0 ; p_{0}\right)=F(p)
$$

and

$$
\chi(q)=\chi_{j}(q), \quad q \in \Gamma_{j},
$$

we have the following formulae for $p$ off $\Gamma$ :

$$
F_{e}\left(p, 0 ; p_{0}\right)=-\frac{1}{2 \pi i} \int_{\Gamma} \frac{\chi(q, 0)\left(F^{\prime}(q)\right)^{2}}{F(p)-F(q)} d q
$$

$$
f_{\varepsilon}(p, 0 ; v)=\frac{(f(p ; v))^{2}}{2 \pi i} \int_{\Gamma} \frac{\chi(q, 0)\left(f^{\prime}(q ; v)\right)^{2} d q}{(f(q ; v))^{2}[f(q ; v)-f(p ; v)]}
$$

$\chi(q, 0)$ is to be regarded as a local reciprocal differential in the above formulae.

We have

$$
F\left(p, \varepsilon ; p_{0}\right)-F\left(p, 0 ; p_{0}\right)=\varepsilon F_{\varepsilon}\left(p, 0 ; p_{0}\right)+o(\varepsilon)
$$

uniformly in any domain whose closure does not meet an interior point of an arc 
$\Gamma_{j}^{-}$(that is, if $p$ varies in such a way that it is bounded away from the lower edge of any slit, except that it is allowed to tend to a vertex of $\Gamma)$ and

$$
f(p, \varepsilon ; v)-f(p, 0 ; v)=\varepsilon f_{e}(p, 0 ; v)+o(\varepsilon)
$$

uniformly in any region bounded away from $\Gamma$.

The first variations of $F$ and $f$ are nontrivial unless $\chi$ vanishes identically.

THEOREM 2.2. Suppose in addition to the hypotheses of Theorem 2.1 that $\chi_{j}(p, \varepsilon)$ is of class $C^{\infty}$ in $\varepsilon$ for $p$ on the closure of $\Gamma_{j}, \varepsilon$ on an interval I about zero, and that the representative $r_{j}(\tau, \varepsilon)$ satisfies the strengthened Höldercondition

$$
\left|\frac{r_{j}\left(\tau, \varepsilon_{2}\right)-r_{j}\left(\tau, \varepsilon_{1}\right)}{\varepsilon_{2}-\varepsilon_{1}}\right|<\left\{\begin{array}{l}
A\left|\tau-\tau\left(a_{j}\right)\right|^{\mu}, \\
A\left|\tau-\tau\left(b_{j}\right)\right|^{\mu},
\end{array} \quad \mu>0,\right.
$$

with $A$ independent of $\varepsilon_{1}, \varepsilon_{2}$ for $p$ within $D_{j}$ and $\varepsilon_{1}, \varepsilon_{2}$ on $I$.

Then $F\left(p, c ; p_{0}\right)$ and $f(p, c ; v)$ are of class $C^{\infty}$ in $c$ for $c$ on any closed subinterval of $I$.

We should remark that the regularity conditions imposed on the particular representative $r_{j}$ of $\chi_{j}$ are easily seen to be independent of the unformizer.

In succeeding sections, we merely verify that for our variations of $Q$-polygons, the functional $\chi_{j}(p, \varepsilon)$ satisfies the hypotheses of Theorems 2.1 and 2.2. Then we calculate $\chi_{j}$ in terms of suitable local parameters and substitute in (2.2) and (2.3).

3. Analytic properties of the functional $\chi$. This section is devoted to showing that the functionals $\chi_{j}$ used in passing from $R(T, Q)$ to $R(T, \tilde{Q})$ satisfy the necessary smoothness conditions for applying the theorems of \$2. The work will be of a rather computational nature.

We work with a representative $r_{j}(\tau, \varepsilon)$ of the sewing deformation in terms of a suitable local parameter $\tau$. A convenient choice for $\tau$ on $\Gamma_{j}$ is the $Q$-distance of $p$ from $a_{j}$, denoted by $\lambda(p)$ and defined by

$$
\lambda(p)= \begin{cases}\int_{a_{j}}^{z} \frac{\sqrt{Q(t)}}{t} d t, & z \in I_{j}, \\ -\int_{a_{j}^{\prime}}^{z^{\prime}} \frac{\sqrt{Q(t)}}{t} d t, & z^{\prime} \in I_{j}^{\prime},\end{cases}
$$

where $z$ and $z^{\prime}$ are the points lying under $p$, and the branches are chosen to be positive on $I_{j}$ and $I_{j}^{\prime}$.

Since $Q$ does not vanish on the interior of $I_{j}$ and $I_{j}^{\prime}$, it is clear that $\lambda(p)$ may 
be taken as a local parameter on $\Gamma_{j}$, and also that $\Gamma_{j}$ is analytic in the parameter $\lambda$ with a definite tangent at the endpoints; indeed the local parameter maps $\Gamma_{j}$ onto a segment of the positive real axis. We see easily, too, that we can use $\lambda(p)$ to define a parametric patch $\mathscr{D}_{j}$ about the interior of $\Gamma_{j}$ which meets the endpoints of $\Gamma_{j}$ at a positive angle.

Now let us calculate $r_{j}(\lambda, \varepsilon)$. The point $p$ on $\Gamma_{j}^{-}$is re-identified with a point $\tilde{p}(\varepsilon)$ on $\Gamma_{j}^{+}$, with a $Q$-distance $\lambda(\tilde{p}(\varepsilon))$ from $a_{j}$, given by

$$
\lambda(\tilde{p}(\varepsilon))=-\int_{a_{j}^{\prime}}^{\zeta(z, \varepsilon)} \frac{\sqrt{Q(t)}}{t} d t
$$

where $\zeta(z, \varepsilon)$ is given by the relation

$$
\int_{a_{j}^{\prime}}^{\zeta(z, \varepsilon)} \sqrt{Q(t, \varepsilon)} \frac{d t}{t}=-\int_{a_{j}}^{z} \sqrt{Q(t, \varepsilon)} \frac{d t}{t},
$$

$z=z(p)$ of course being the point on $I_{j}$ over which $p$ lies. Then $r_{j}(\lambda, \varepsilon)$ is defined by the equation

$$
\lambda(\tilde{p}(\varepsilon))=\lambda+\varepsilon r_{j}(\lambda, \varepsilon), \quad \varepsilon \neq 0,
$$

and we define

$$
r_{j}(\lambda, 0)=\lim _{\varepsilon \rightarrow 0} r_{j}(\lambda, \varepsilon)
$$

provided this limit exists. Combining these remarks with (3.1) and (3.2) we have, for $\varepsilon \neq 0$, the equation

$$
\begin{aligned}
r_{j}(\lambda, \varepsilon) & =\frac{1}{\varepsilon}(\lambda(\tilde{p}(\varepsilon))-\lambda) \\
& =\frac{-1}{\varepsilon}\left[\int_{a_{j}^{\prime}}^{\zeta(z, \varepsilon)} \frac{\sqrt{Q(t)}}{t} d t+\int_{a_{j}}^{z} \frac{\sqrt{Q(t)}}{t} d t\right], \quad \varepsilon \neq 0,
\end{aligned}
$$

and, if we let $\tilde{z}(z, \varepsilon)$ denote the point on $I_{j}$ which is identified with $\zeta(z, \varepsilon)$ under the $Q$-metric, we have the alternate expression

$$
r_{j}(\lambda, \varepsilon)=\frac{1}{\varepsilon}\left[\int_{a_{j}}^{z} \frac{\sqrt{Q(t)}}{t} d t-\int_{a_{j}}^{\tilde{z}(z, e)} \frac{\sqrt{Q(t)}}{t} d t\right], \quad \varepsilon \neq 0 .
$$

It is clear that the regularity properties of $r_{j}$ depend on the nature of the function $\zeta(z, \varepsilon)$ defined by (3.2), which we now proceed to study. Since a lemma would be rather cumbersome let us just proceed with our development.

We are going to assume now that $Q(t, \varepsilon)$ is analytic in $\varepsilon$ for all $t$ on the unit circumference.

It is easy now to see that $\zeta(z, \varepsilon)$ and, hence, $\tilde{z}(z, \varepsilon)$ are analytic in $\varepsilon$ and $z$ for $z$ on the interior of $I_{j}$. This follows by a simple implicit function argument 
applied to equation (3.2), making use of the fact that $Q(t, \varepsilon)$ does not vanish on the interior of $I_{j}^{\prime}$. This enables us to take care of the problem of defining $r_{j}(\lambda, 0)$, for we see that $r_{j}(\lambda, 0)$ is just the derivative with respect to $\varepsilon$, at $\varepsilon=0$, of the term in parentheses in (3.3). We have in fact

$$
r_{j}(\lambda, 0)=-(\sqrt{Q(z)} / z) \tilde{z}_{\varepsilon}(z, 0), \quad z \in I_{j} .
$$

Now let us introduce the terminology

$$
A(\varepsilon, \zeta)=\int_{a_{j}^{\prime}}^{\zeta} \frac{\sqrt{Q(t, \varepsilon)}}{t} d t, \quad B(\varepsilon, z)=-\int_{a_{j}}^{z} \frac{\sqrt{Q(t, \varepsilon)}}{t} d t,
$$

and consider (3.2) in the form

$$
A(\varepsilon, \zeta)=B(\varepsilon, z)
$$

regarded as an equation for the determination of $\zeta$. We concentrate on $z$ near $a_{j}$. We can expand both sides of (3.5) in power series, obtaining

$$
\sum_{\nu=N^{\prime}}^{\infty} \alpha_{\nu}(\varepsilon)\left(\zeta-a_{j}^{\prime}\right)^{\nu}=\sum_{\sigma=N}^{\infty} \beta_{\sigma}(\varepsilon)\left(z-a_{j}\right)^{\sigma}
$$

with $N, N^{\prime}$ nonnegative integers (since $Q$ vanishes of even order when it vanishes on the circumference) independent of $\varepsilon$, and both series having positive radii of convergence which can be regarded as independent of $\varepsilon$ for $\varepsilon$ small. The $\alpha_{\nu}$ and $\beta_{\sigma}$ are analytic functions of $\varepsilon$, and since the order of zeroes of $Q(t, \varepsilon)$ is invariant, $\beta_{N}(\varepsilon)$ and $\alpha_{N^{\prime}}(\varepsilon)$ are bounded away from zero.

Set $t$ equal to the common value of both sides of $\left(3.5^{\prime}\right)$. We obtain an expansion

$$
\zeta-a_{j}^{\prime}=\sum_{\nu=1}^{\infty} \gamma_{\nu}(\varepsilon) t^{\nu / N^{\prime}}, \quad \gamma_{1}(\varepsilon) \neq 0,
$$

and inserting this into the other member of $\left(3.5^{\prime}\right)$ gives us

$$
\zeta-a_{j}^{\prime}=\sum_{\nu=1}^{\infty} \delta_{\nu}(\varepsilon)\left(z-a_{j}\right)^{\nu N / N^{\prime}}, \quad \delta_{1}(\varepsilon) \neq 0 .
$$

Now observe that $\tilde{z}=\tilde{z}(z, \varepsilon)$ is by definition found from $\zeta$ by putting $\varepsilon=0$ in (3.5) and solving for $z$, so that the same computations that led to (3.6) will give us

$$
\zeta-a_{j}^{\prime}=\sum_{\sigma=1}^{\infty} \delta_{\sigma}(0)\left(\tilde{z}-a_{j}\right)^{\sigma N / N^{\prime}}, \quad \delta_{1}(0) \neq 0 .
$$

We can invert the series (3.7) to obtain

$$
\tilde{z}-a_{j}=\sum_{\sigma=1}^{\infty} d_{\sigma}\left(\zeta-a_{j}^{\prime}\right)^{\sigma N^{\prime} / N}, \quad d_{1} \neq 0,
$$

and if we now use (3.7) to eliminate $\zeta-a_{j}^{\prime}$ we find that $z$ is related to $z$ by a 
formula of the form

$$
\tilde{z}-a_{j}=\sum_{\nu=1} C_{\nu}(\varepsilon)\left(z-a_{j}\right)^{\nu}
$$

where the $C_{\nu}(\varepsilon)$ are analytic at $\varepsilon=0$, and $C_{1}(\varepsilon)$ is bounded away from zero and infinity.

We can use (3.8) to express $r_{j}(\lambda, \varepsilon)$ as a series in $\lambda$ for small $\lambda$. Indeed, setting

$$
\lambda=\int_{a_{j}}^{z} \frac{\sqrt{Q(t)}}{t} d t=\sum_{\sigma=N+1}^{\infty} \beta_{\sigma}\left(z-a_{j}\right)^{\circ}
$$

gives us an expansion

$$
z-a_{j}=\sum_{\sigma=1}^{\infty} k_{\sigma} \lambda^{\sigma / N}, \quad k_{1} \neq 0,
$$

and using this in (3.8) we have

$$
\tilde{z}-a_{j}=\sum_{\nu=1}^{\infty} d_{\nu}(\varepsilon) \lambda^{\nu / N}, \quad d_{1}(\varepsilon) \neq 0 .
$$

Now observe that $B(0, \tilde{z})$ can be computed by using (3.10) in (3.9), yielding an expansion

$$
B(0, \tilde{z}(z, \varepsilon))=\sum_{\nu=N}^{\infty} e_{\nu}(\varepsilon) \lambda^{\nu / N}
$$

Now go back to (3.3), observe that it can be written in the form

$$
\varepsilon r_{j}(\lambda, \varepsilon)=B(0, z)-B(0, \tilde{z}(z, \varepsilon))=\lambda-B(0, \tilde{z}(z, \varepsilon)),
$$

and we have

$$
\varepsilon r_{j}(\lambda, \varepsilon)=\lambda-\sum_{\nu=N}^{\infty} e_{\nu}(\varepsilon) \lambda^{\nu / N},
$$

valid for sufficiently small $\lambda$.

We now verify the requisite smoothness properties of $r_{j}(\lambda, \varepsilon)$.

LEMMA 3.1. Let $Q(t, \varepsilon)$ be analytic in $\varepsilon$ for $|t|=1$. Then $r_{j}(\lambda, \varepsilon)$ is analytic in $\varepsilon$ for $p$ on the closure of $\Gamma_{j}$, and $r_{j}(\lambda, \varepsilon)$ satisfies (2.1) with $\mu=1$.

Proof. First, assume $p$ not an endpoint of $\Gamma_{j}$. Then by (3.3) we see that $r_{j}(\lambda, \varepsilon)$ is analytic in $\varepsilon$ except perhaps at $\varepsilon=0$. We can regard $\varepsilon$ as being complex-valued, however, in the above discussion, and varying throughout a small neighborhood of the origin. Since we know the limit $r_{j}(\lambda, 0)$ exists as a finite value, the singularity at $\varepsilon=0$ is removable.

Now let $p$ be $a_{j}$, so that the expansion (3.12) is valid. By precisely the same argument as above, $r_{j}(\lambda, \varepsilon)$ is analytic in $\varepsilon$ at $a_{j}$. 
For $p$ sufficiently near $a_{j}$, expansion (3.12) is valid. The coefficients must evidently all vanish at least of order one at $\varepsilon=0$, and we see that $r_{j}(\lambda, \varepsilon)$ has a uniformly bounded derivative with respect to $\lambda$ for small $\lambda$. This is more than enough to prove the Hölder-condition (2.1) at $a_{j}$.

One could of course obtain a series like (3.12) near $b_{j}$, in terms of powers of $\lambda-\lambda\left(b_{j}\right)$, so there is no difficulty about the other endpoint.

LEMMA 3.2 If $Q(t, \varepsilon)$ is analytic in $\varepsilon$ for $|t|=1$, then $r_{j}(\lambda, \varepsilon)$ is analytic in $\lambda$ for $p$ on the interior of $\Gamma_{j}$, and bounded independently of $\varepsilon$ on the closure of $\Gamma_{j}$.

Proof. On the interior of $\Gamma_{j}$, analyticity with respect to $\lambda$ and with respect to $z$ are the same thing. Since we have verified that $\tilde{z}(z, \varepsilon)$ is analytic in $z$, (3.3) and (3.4) verify our conclusion in the cases $\varepsilon \neq 0$ and $\varepsilon=0$, respectively.

LEMMA 3.3. If $Q(t, \varepsilon)$ is analytic in $\varepsilon$ for $|t|=1$, then $r_{j}(\lambda, \varepsilon)$ satisifes the second Hölder-condition, (2.4), at $a_{j}$ and $b_{j}$.

Proof. Applying the Law of the Mean separately to real and imaginary parts we see that it suffices to show that the partial derivative of $r_{j}(\lambda, \varepsilon)$ with respect to $\varepsilon$ satisfies a Hölder-condition of the form (2.1).

We can use the series (3.12) for $p$ near $a_{j}$. We have already pointed out that the coefficients all have at least a simple zero at $\varepsilon=0$, thus

$$
r_{j}(\lambda, \varepsilon)=\sum_{\nu=N}^{\infty} e_{\nu}^{*}(\varepsilon) \lambda^{\nu / N},
$$

the $e_{\nu}^{*}$ analytic at $\varepsilon=0$, and we can differentiate term-by-term to obtain, formally,

$$
\frac{\partial r_{j}(\lambda, \varepsilon)}{\partial \varepsilon}=\sum e_{\nu}^{* \prime}(\varepsilon) \lambda^{\nu / N}
$$

Since $r_{j}(\lambda, \varepsilon)$ is biholomorphic in the variables $\varepsilon, \lambda^{1 / N}$ at $\varepsilon=\lambda=0$, this series is convergent for sufficiently small $\varepsilon$ and $\lambda$, and $\partial r_{j}(\lambda, \varepsilon) / \partial \varepsilon$ is bounded by some fractional power of $\lambda$.

One verifies the condition at the endpoint $b_{j}$ by using a series like (3.12) in powers of $\lambda-\lambda\left(b_{j}\right)$.

This completes the proof of the necessary smoothness conditions on $r_{j}(\lambda, \varepsilon)$. For possible future reference, the author would like to point out that the conclusions of this section hold also if $Q(t, \varepsilon)$ is a piecewise analytic function of the form $Q(t, \varepsilon)=Q_{j}(t, \varepsilon), t \in I_{j}$ or $I_{j}^{\prime}$ where each $Q_{j}(t, \varepsilon)$ is positive on $I_{j} \cup I_{j}^{\prime}$, and analytic in a region including the closure of $I_{j} \cup I_{j}^{\prime}$. We can relax the conditions on $Q_{j}$ further, requiring only that $Q_{j}(t, \varepsilon)$ have a convergent expansion in fractional powers, convergent in some sector about the endpoints of arcs. 
4. The variational formula for $f$ and $F$. These are found by finding a representative $r_{j}(\tau, \varepsilon)$ for $\chi_{j}(p, \varepsilon)$, and carrying out the integration (2.2) in terms of $\tau$.

We use the local parameter $\lambda$ of $\S 3$, and explicitly compute $r_{j}(\lambda, 0)$, which by (3.4) can be done once we know $\tilde{z}_{\varepsilon}(z, 0)$. Differentiating (3.5) with respect to $\varepsilon$ and setting $\varepsilon=0$ we get

$$
-2 \zeta_{\varepsilon}(z, 0)=\frac{\zeta}{\sqrt{Q(\zeta)}}\left[\int_{a_{j}}^{z} \frac{Q_{\varepsilon}(t, 0)}{\sqrt{Q(t)}} \frac{d t}{t}+\int_{a_{j}}^{\zeta} \frac{Q_{\varepsilon}(t, 0)}{\sqrt{Q(t)}} \frac{d t}{t}\right]
$$

where we understand $Q(\zeta)=Q(\zeta, 0)$, etc. Now $\tilde{z}$ is related to $\zeta$ by the equation

$$
B(0, \tilde{z})=A(0, \zeta)
$$

which upon differentiation yields

$$
\tilde{z}_{\varepsilon}=A_{\zeta} \zeta_{\varepsilon} / B_{\tilde{z}}=-\frac{\sqrt{Q(\zeta)}}{\zeta} \frac{\tilde{z}}{\sqrt{Q(\tilde{z})}} \zeta_{\varepsilon},
$$

which for $\varepsilon=0$ is just

$$
\tilde{z}_{\varepsilon}(z, 0)=-\frac{\sqrt{Q(\zeta)}}{\zeta} \frac{z}{\sqrt{Q(z)}} \zeta_{\varepsilon}(z, 0) .
$$

Now we use the data (4.1), (4.2) in (3.4) to obtain

$$
-2 r_{j}(\lambda, 0)=\int_{a_{j}}^{z} \frac{Q_{\varepsilon}(t, 0)}{\sqrt{Q(t)}} \frac{d t}{t}+\int_{a_{j}^{\prime}}^{\zeta(z, 0)} \frac{Q_{\varepsilon}(t, 0)}{\sqrt{Q(t)}} \frac{d t}{t} .
$$

Now when $\varepsilon=0$, (3.5) reduces to (1.1), so we have finally

$$
-2 r_{j}(\lambda, 0)=\int_{a_{j}}^{z} \frac{Q_{\varepsilon}(t, 0)}{\sqrt{Q(t)}} \frac{d t}{t}+\int_{a_{j}^{\prime}}^{z^{\prime}} \frac{Q_{\varepsilon}(t, 0)}{\sqrt{Q(t)}} \frac{d t}{t},
$$

where $z \in I_{j}$ and $z^{\prime} \in I_{j}^{\prime}, z, z^{\prime}$ related by (1.1).

Now we must change from the local parameter $\lambda$ to a local parameter which provides a workable variable of integration. So, for $p$ on the interior of $\Gamma_{j}$, we define the local parameter $z=z(p)$ by simple projection onto $I_{j}$. This of course gives us a different representation, call it $r_{j}^{*}(z, 0)$, for the re-identification function $\chi(p, 0)$. Since $\chi(p, 0)$ is a local reciprocal differential in the parametric patch $\mathscr{D}_{j}$ (according to the general theory discussed in [2]) we have

$$
r_{j}^{*}(z, 0)=r_{j}(\lambda, 0) \frac{d z}{d \lambda}=\frac{z}{\sqrt{Q(z)}} r_{j}(\lambda, 0) .
$$


We can now write down the variational formula. Let us replace $p, q$ in (2.2) by their projections $w$ and $z$, respectively, in the plane. Introduce the auxiliary function

$$
X_{j}(z)=\int_{a_{j}}^{z} \frac{Q_{\varepsilon}(t, 0)}{\sqrt{Q(t)}} \frac{d t}{t}+\int_{a_{j}^{\prime}}^{z^{\prime}} \frac{Q_{\varepsilon}(t, 0)}{\sqrt{Q(t)}} \frac{d t}{t},
$$

and finally, for convenience, set

$$
E(t)=Q_{\varepsilon}(t, 0)
$$

Using (4.3) and (4.4), (2.2) becomes

$$
F_{e}(w, 0)=-\frac{1}{4 \pi i} \sum_{j} \int_{I_{j}} \frac{z}{\sqrt{Q(t)}} \frac{\left(F^{\prime}(z)\right)^{2} X_{j}(z)}{F(w)-F(z)} d z
$$

and (2.3) becomes

$$
f_{e}(w, 0 ; v)
$$

$$
=\frac{(f(w ; v))^{2}}{4 \pi i} \sum_{j} \int_{I_{j}} \frac{z}{\sqrt{Q(z)}} \frac{\left(f^{\prime}(z ; 0)\right)^{2} X_{j}(z)}{(f(z ; v))^{2}[f(w ; v)-f(z ; v)]} d z .
$$

We can replace (4.5) and (4.6) by similar formulas which involve the arcs $I_{j}$ and $I_{j}^{\prime}$ in a symmetric manner. Let $b$ be the common endpoint of an abutting pair of indentified arcs (there is certainly at least one such point) and define a further auxiliary function $T(z)$ on $|z|=1$ by

$$
T(z)=\int_{b}^{z} \frac{\sigma(t) E(t)}{t \sqrt{Q(t)}} d t,
$$

where $\sigma(t)$ is 1 or -1 according as $t$ is, respectively, on an arc $I_{j}$ or an arc $I_{j}^{\prime}, \sqrt{Q(t)}$ is nonnegative, and we integrate over the unit circle in the positive sense.

Suppose now that $g(z)$ is any function integrable over the unit circle which takes the same value at identified points. Then

$$
\begin{aligned}
\int_{I_{j}} g(z) \frac{z}{\sqrt{Q(z)}}\left(F^{\prime}(z)\right)^{2} X_{j}(z) d z & \\
= & \int_{I_{j}} g(z) \frac{z}{\sqrt{Q(z)}}\left(F^{\prime}(z)\right)^{2}\left(T(z)-T\left(a_{j}\right)\right) d z \\
& -\int_{I_{j}} g(z) \frac{z}{\sqrt{Q(z)}}\left(F^{\prime}(z)\right)^{2}\left(T\left(z^{\prime}\right)-T\left(a_{j}^{\prime}\right)\right) d z
\end{aligned}
$$


In the second integral appearing on the right-hand side of (4.7) we change the variable of integration to $z^{\prime}$. As $z$ traverses the arc $I_{j}=\left(a_{j}, b_{j}\right)$ from $a_{j}$ to $b_{j}, z^{\prime}$ describes $I_{j}^{\prime}$ in the direction $a_{j}^{\prime}$ to $b_{j}^{\prime}$. By hypothesis $g\left(z^{\prime}\right)=g(z)$, the relation $F(z)=F\left(z^{\prime}\right)$ implies

$$
\left(F^{\prime}(z)\right)^{2}=\left(F^{\prime}\left(z^{\prime}\right)\right)^{2}\left(d z^{\prime} / d z\right)^{2}
$$

and relation (1.1) gives

$$
\frac{z}{\sqrt{Q(z)}}=-\frac{z^{\prime}}{\sqrt{Q\left(z^{\prime}\right)}} \frac{d z}{d z^{\prime}}=\sigma\left(z^{\prime}\right) \frac{z^{\prime}}{\sqrt{Q\left(z^{\prime}\right)}} \frac{d z}{d z^{\prime}} .
$$

Thus we can rewrite (4.7) as follows:

$$
\begin{aligned}
\int_{I_{j}} g(z) \frac{z}{\sqrt{Q(z)}}\left(F^{\prime}(z)\right)^{2} X_{j}(z) d z & \\
= & \left(\int_{a_{j}}^{b_{j}}+\int_{b_{j}^{\prime}}^{a_{j}^{\prime}}\right) g(z) T(z) \frac{z}{\sqrt{Q(z)}}\left(F^{\prime}(z)\right)^{2} \sigma(z) d z \\
& +\left(T\left(a_{j}^{\prime}\right)-T\left(a_{j}\right)\right) \int_{I_{j}} g(z) \frac{z}{\sqrt{Q(z)}}\left(F^{\prime}(z)\right)^{2} d z
\end{aligned}
$$

To simplify (4.8) further, we prove the following lemma:

LeMma 4.1. For all $j, T\left(a_{j}^{\prime}\right)-T\left(a_{j}\right)=0$.

Proof. Proceeding from $b$ counterclockwise around the unit circle, mark off the points $w_{0}(=b), w_{1}, w_{2}, \ldots$, which are either left endpoints of arcs $I_{j}$ or right endpoints of arcs $I_{j}^{\prime}$. If $w_{k}$ is the left endpoint of $I_{j}$ let $w_{k}^{\prime}$ be the right endpoint of $I_{j}^{\prime}$, and if $w_{k}$ is the right endpoint of $I_{j}^{\prime}$ let $w_{k}^{\prime}$ be the left endpoint of $I_{j}$.

Obviously $T\left(w_{0}^{\prime}\right)-T\left(w_{0}\right)=0$. Assume inductively that

$$
T\left(w_{k}^{\prime}\right)=T\left(w_{k}\right), \quad k \leqslant n-1 .
$$

Now the $\operatorname{arc}\left(w_{n-1}, w_{n}\right)$ is either $I_{j}$ or an $I_{j}^{\prime}$. In either case we have, by the inductive hypothesis,

$$
\begin{aligned}
T\left(w_{n}^{\prime}\right)-T\left(w_{n}\right) & =\left(T\left(w_{n}^{\prime}\right)-T\left(w_{n-1}^{\prime}\right)\right)-\left(T\left(w_{n}\right)-T\left(w_{n-1}\right)\right) \\
& = \pm X_{j}\left(b_{j}\right)
\end{aligned}
$$

which vanishes because our sewing variation leaves endpoints of arcs fixed.

Then (4.8) reduces to 


$$
\begin{aligned}
\int_{I_{j}} g(z) \frac{z}{\sqrt{Q(z)}}\left(F^{\prime}(z)\right)^{2} X_{j}(z) d z \\
=\left(\int_{a_{j}}^{b_{j}}+\int_{b_{j}^{\prime}}^{a_{j}^{\prime}}\right) g(z) T(z) \frac{z}{\sqrt{Q(z)}} \sigma(z) d z .
\end{aligned}
$$

The functions $F(w)-F(z)$ and $(f(z ; v))^{2}[f(w ; v)-f(z ; v)]$ are invariant when $z$ on $I_{j}$ is replaced by $z^{\prime}$ on $I_{j}^{\prime}$, so the preceding discussion applies to the integrals (4.5), (4.6) and (4.6'). Summation over $j$ yields an integral over the unit circumference. We can get rid of the function $\sigma$ by re-interpreting $\sqrt{Q(z)}$ to be positive on $\operatorname{arcs} I_{j}$ and negative on $\operatorname{arcs} I_{j}^{\prime}$.

We bring our considerations together in a theorem.

THEOREM 4.1. Let $\Re(T, Q(\varepsilon))$ be a family of $Q$-polygons formed from the same system $T$ of identified arcs and identified endpoints of those arcs, and let $Q(z, \varepsilon)$ be analytic in $\varepsilon$ for $\varepsilon$ on an interval I about zero.

Then the function $f(w, \varepsilon ; v)$ which maps $R(T, Q(\varepsilon))$ conformally onto $\mathcal{C}$ and has its pole at a fixed vertex $v$ of $T$, with $f(0, v)=0$ and $f^{\prime}(0, v)=1$, has the variational formula

$$
\begin{aligned}
& f_{\varepsilon}(w, 0 ; v) \\
& =\frac{(f(w ; v))^{2}}{4 \pi i} \int_{|z|=1} \frac{z}{\sqrt{Q(z)}} \frac{\left(f^{\prime}(z ; v)\right)^{2} S(z) d z}{(f(z ; v))^{2}[f(w ; v)-f(z ; v)]}
\end{aligned}
$$

where

$$
S(z)=\int_{b}^{z} \frac{Q_{\varepsilon}(t, 0)}{\sqrt{Q(t)}} d t .
$$

We take $\sqrt{Q(z)}$ positive or negative according as $z$ is on an arc $I_{j}$ or an arc $I_{j}^{\prime}$. In (4.10) $b$ is the common endpoint of an abutting pair of identified arcs.

$f(w, c: v)$ is of class $C^{\infty}$ in $c$ on any closed subinterval of $I$.

We have

$$
f(w, \varepsilon ; v)-f(w ; v)=\varepsilon f_{\varepsilon}(w, 0 ; v)+o(\varepsilon)
$$

uniformly in domains bounded away from the unit circumference.

Similar remarks apply to the function $F(w, \varepsilon)$, which maps $\Re(T, Q(\varepsilon))$ onto $\mathcal{C}$, and has at $w=0$ a simple pole with residue 1 and zero translation. For $F(w, \varepsilon)$, we have

$$
F_{\varepsilon}(w, 0)=-\frac{1}{4 \pi i} \int_{|z|=1} \frac{z}{\sqrt{Q(z)}} \frac{\left(F^{\prime}(z)\right)^{2} S(z)}{F(w)-F(z)} d z .
$$


We remark that the formulae (4.9), (4.10) are the same as those obtained by Schaeffer and Spencer [3, p. 173] for the extremal schlicht functions mapping the unit disc onto the plane slit along a single analytic arc. In their case, $T$ consists of a single pair $I_{1}, I_{1}^{\prime}$ of identified arcs which, however, are allowed to have variable endpoints. Thus our results overlap but do not contain those of [3].

5. A necessary condition for the first variation to be trivial. If the first variation vanishes identically it is not, for most purposes, a useful approximation to the actual increment of the functional under consideration. Thus we inquire when the integral (4.9) can vanish identically. We will have to make an excursion into the calculus of higher-order differentials on Riemann surfaces. For background we refer the reader to [4, pp. 76-87].

LEMMA 5.1. Let $\pi$ be a Riemann surface conformally equivalent to $\mathcal{C}$, and let 2 be a quadratic differential of $\Re$ with a pole at $p_{0}$. Let $F(p)$ be the function which maps $\Re$ conformally onto $\mathcal{C}$, with a pole of unit residue at $p_{0}$.

Then

$$
\mathcal{2}(p)=R(F(p))\left(F^{\prime}(p)\right)^{2}
$$

for some rational function $R$.

Proof. For simplicity let us first assume $p_{0}$ is the only pole of 2. By $[4, \mathrm{p}$. 78] 2 has at least four poles, multiplicity counted. Now the functional $(F(p))^{n}\left(F^{\prime}(p)\right)^{2}$ is a quadratic differential having a pole at $p_{0}$ of order $n+4$ as its only singularity. Thus we can choose the coefficients of a polynomial $P$ so that $2(p)-P(F(p))\left(F^{\prime}(p)\right)^{2}$ is a quadratic differential with at most three poles, which then must vanish identically.

In general, 2 has finitely many poles away from $p_{0}$, say at points $p_{k}$ with repetitions according to multiplicity. We form the new quadratic differential

$$
\mathcal{Q}_{1}(p)=2(p) \prod_{k}\left(F(p)-F\left(p_{k}\right)\right)
$$

and treat $\mathscr{Q}_{1}$ as in the preceding paragraph.

Lemma 5.2. Let $\Re(T, Q)$ be a $Q$-polygon, $Q$ meromorphic on $\mathcal{C}$. Suppose there is another function $Q^{*}$, meromorphic on $\mathcal{C}$, such that

$$
\int_{a_{j}}^{z} \sqrt{Q^{*}(t)} \frac{d t}{t}=-\int_{a_{j}^{\prime}}^{z^{\prime}} \sqrt{Q^{*}(t)} \frac{d t}{t}, \quad z \in I_{j}, z^{\prime} \in I_{j}^{\prime},
$$

where $z$ and $z^{\prime}$ are as in (1.1).

Assume also that the quotient $Q^{*} / Q$ does not reduce to a constant.

Then the mapping function $f(w)$ is algebraic.

Proof. We can use (5.1) just as in $\S 1$ to form a Riemann surface $\mathcal{R}\left(T, Q^{*}\right)$ 
corresponding to the same arcs and identification system. We have to observe here that $\mathscr{R}(T, Q)$ is not changed if we subdivide arcs $I_{j}, I_{j}^{\prime}$, consistently with (1.1), so we can suppose all zeroes of $Q(z)$ or $Q^{*}(z)$ on the unit circle are found among endpoints of the arcs.

Now let $F_{Q}(z)$ and $F_{Q^{*}}(z)$ be, respectively, the conformal mappings of $\Re(T, Q)$ and $\Re\left(T, Q^{*}\right)$ onto $C$ having at the point $p_{0}$ lying over the origin a simple pole with residue unity. $\left(F_{Q}\right.$ is of course just the reciprocal of $f$.) Let $\tau(p)$ be the local parameter system on $\Re(T, Q)$ defined in $\S 1$. We observe that $F_{Q^{*}}$ is regular on $\Re(T, Q)$ save perhaps at vertices, for we can simply invert equations for $z$ in terms of the uniformizers on the respective surfaces. Since $F_{Q^{*}}$ is single-valued on $\Re(T, Q)$, we see that these singularities are removable; thus $F_{Q^{*}}$ is regular on $\Re(T, Q)$. By composing mappings to $\mathcal{C}$ we see that $F_{Q^{*}}$ is a bilinear function of $F_{Q}$ and, because of the normalization,

$$
F_{Q}(p)=F_{Q^{*}}(p) \text {. }
$$

Now as we observed at the end of $\S 1, Q$ and $Q^{*}$ induce quadratic differentials 2 and $2^{*}$ on $\Re(T, Q)$ and $\Re\left(T, 2^{*}\right)$, respectively. Applying Lemma 5.1 on $\Re(T, Q)$ and $R\left(T, Q^{*}\right)$ and then setting

$$
F_{Q}(p)=F_{Q^{*}}(p)=F(p)
$$

we get the pair of equations

$$
\begin{gathered}
\mathcal{Q}(p)=R(F(p))\left(F^{\prime}(p)\right)^{2}, \\
\mathcal{Q}^{*}(p)=R^{*}(F(p))\left(F^{\prime}(p)\right)^{2} .
\end{gathered}
$$

Divide one equation by the other, then let $p$ lie over a point of $|z|<1$, so that $\mathcal{L}(p)$ and $\mathcal{2}^{*}(p)$ are represented by $Q(z) / z^{2}$ and $Q^{*}(z) / z^{2}$. We have

$$
Q(z) / Q^{*}(z)=R(F(z)) / R^{*}(F(z))
$$

showing that $F(z)$ is algebraic.

Now we can show that, normally, the first variation does not vanish identically.

THEOREM 5.1. Let $\Re(T, Q(\varepsilon))$ be a family of $Q$-polygons formed with the same system $T$ of arcs and identifications. Suppose that $f_{\varepsilon}(w, 0)$ vanishes for $|w|<1$. Suppose $Q(\varepsilon, z)$ is meromorphic on $C$.

Then either $Q_{\varepsilon}(z, 0) / Q(z)$ reduces to a constant, or $f(w)$ is algebraic.

Proof. We have seen (Theorem 2.1) that the sewing functional $\chi(q, 0)$ vanishes identically. In the present context this implies, by (4.3), the equation

$$
\int_{a_{j}}^{z} \frac{Q_{\varepsilon}(t, 0)}{\sqrt{Q(t)}} \frac{d t}{t}=-\int_{a_{j}^{\prime}}^{z^{\prime}} \frac{Q_{\varepsilon}(t, 0)}{\sqrt{Q(t)}} \frac{d t}{t}, \quad z \in I_{j}, z^{\prime} \in I_{j}^{\prime}
$$

(positive roots on both sides). Taking 


$$
Q^{*}=\left(Q_{\varepsilon}(t, 0)\right)^{2} / Q(t)
$$

we see that Lemma 5.2 applies.

Some further light is shed by the following corollary, which is essentially [3, p. 156 (Lemma XXXI)].

COROLLARY 5.1.1. Under the hypotheses of Theorem 5.1, suppose further that

$$
Q(z)=\sum_{k=-N}^{N} B_{k} z^{k},
$$

with $N$ prime.

Then either $Q_{\varepsilon}(z, 0) / Q(z)$ reduces to a constant, or

$$
f(w)=w /\left(1-e^{i \alpha} w\right)\left(1-e^{i \beta_{w}}\right),
$$

$\alpha, \beta$ real.

6. A partial surface variation for the extremal schlicht functions. The extremal schlicht functions for the coefficient body $\mho_{N+1}$ are precisely the functions $f$ which are regular and schlicht in the unit disc, with normalization $f(0)=0, f^{\prime}(0)=1$, which map a $Q$-polygon conformally to $C$ with $Q$ of degree $N$; that is

$$
Q(z)=\sum_{j=-N}^{N} B_{j} z^{j} .
$$

This is the major result obtained by Schaeffer and Spencer [3, p. 120]. Because multiplication of $Q$ by a positive constant does not change the corresponding extremal schlicht function, we may as well write $Q$ in the normalized form

$$
Q(z)=\prod_{k=1}^{m}\left(\frac{\left(z-\zeta_{k}\right)^{2}}{-z \zeta_{k}}\right)^{\mu_{k}} \prod_{\nu=1}^{n}\left(1+\left|z_{\nu}\right|^{2}-\frac{z_{\nu}}{z}-\bar{z}_{\nu} z\right)
$$

where the $\zeta_{k}$ are on the unit circumference and the $z_{v}$ are in the open unit disc. Here; we assume the $\zeta_{n}$ are distinct. We assume $Q(z)$ has at least one zero of modulus one.

It is reasonable to try to express the variation of the mapping functions in terms of the parameters $z_{v}$. Starting with a fixed $Q$, and a consistent tree $T$, we obtain a variation of $\Re(Q, T)$ of the kind studied in preceding sections if we fix the $\zeta_{k}$ and $\mu_{k}$ in (6.1), and vary the $z_{\nu}$ in such a way that we preserve the relations

$$
\int_{I_{j}} \frac{\sqrt{Q(t)}}{t} d t=\int_{I_{j}^{\prime}} \frac{\sqrt{Q(t)}}{t} d t, \quad\left(I_{j}, I_{j}^{\prime} \text { fixed }\right) .
$$

Let us study the mapping function $f\left(w, \zeta_{1}\right)$ which has its pole at $\zeta_{1}$. 
We let $M$ be the number of pairs of identified arcs, and put $z_{\nu}=x_{2 \nu-1}+$ $i x_{2 \nu}, \nu=1,2, \ldots, n$. When we fix the zeroes of $Q$ on the circumference, $Q$ becomes a function of the $2 n$ parameters $x_{k}$, so we write $Q$ as $Q(z$; $\left.x_{1}, x_{2}, \ldots, x_{2 n}\right)$. Equations (6.2) impose $M$ constraints on $2 n$ parameters. Set

$$
H_{j}\left(x_{1}, \ldots, x_{2 n}\right)=\left(\int_{I_{j}}-\int_{I_{j}}\right) \frac{\sqrt{Q\left(t ; x_{1}, \ldots, x_{2 n}\right)}}{t} \frac{d t}{t} .
$$

Then the $H_{j}$ are easily seen to be analytic functions of the $x_{p}$, and if the system of linear equations

$$
\sum_{\nu=1}^{2 n} \frac{\partial H_{j}}{\partial x_{\nu}} \delta x_{\nu}=0, \quad j=1-M
$$

has rank $m(m \leqslant M)$ we can solve the system

$$
H_{j}\left(x_{1}, \ldots, x_{2 n}\right)=0, j=1-M,
$$

for $x_{1}, \ldots, x_{m}$ as analytic functions of $x_{m+1}, \ldots, x_{2 n}$, re-indexing the $x_{v}$ if necessary, so that we obtain a family of admissible $Q$-functions depending smoothly on at least $2 n-M$ real parameters, provided $2 n>M$. By Theorem 4.1 the smoothness of the $Q$-functions implies the smoothness of the corresponding extremal schlicht functions in their dependence on these parameters; thus, if $\left(a_{2}, \ldots, a_{N+1}\right)$ is the point of $\partial \mathcal{V}_{N+1}$ corresponding to $\Re(Q, T), \partial \mathscr{V}_{N+1}$ contains a smooth submanifold of dimension at least $2 n-$ $M$ about this point. We can augment the dimension of this smooth submanifold by one more, by considering the function $f(w, \zeta)$ which has its pole at $\zeta$ on the circumference, with $\zeta$ no longer restricted to be a vertex of the polygon. We have

$$
f(w, \zeta)=f\left(\zeta, \zeta_{1}\right) f\left(w, \zeta_{1}\right) /\left[f\left(w, \zeta_{1}\right)-f\left(\zeta, \zeta_{1}\right)\right] .
$$

The tangent manifold for $f\left(w, \zeta_{1}\right)$ is generated by the variations obtained by varying one of the $x_{j}$, subject to the constraints $(6.3),\left(6.3^{\prime}\right)$. It is easy to check that

$$
\partial Q(z) / \partial x_{j}=Q(z) x_{j} p_{j}(z)
$$

with

$$
p_{j}(z)= \begin{cases}\frac{2 x_{j}-(z+1 / z)}{1+\left|z_{j}\right|^{2}-z_{j} / z-\bar{z}_{j} z}, & j \text { odd }, \\ \frac{2 x_{j}+i(z-1 / z)}{1+\left|z_{j}\right|^{2}-z_{j} / z-\bar{z}_{j} z}, & j \text { even. }\end{cases}
$$

The corresponding function $S$ of (4.10) is 


$$
S_{j}(z)=x_{j} \int_{b}^{z} \frac{\sqrt{Q(t) p_{j}(t)}}{t} d t
$$

We obtain the tangent manifold by setting

$$
S(z)=\sum_{k=1}^{2 n} C_{k} S_{k}(z)
$$

with real coefficients $C_{k}$ subject to the constraints

$$
\sum_{k=1}^{2 n} C_{k} \int_{I_{j} \cup I_{j}^{\prime}} S_{k}(t) d t=0, \quad j=1,2, \ldots, m,
$$

where we interpret $\sqrt{Q(t)}$ to be positive on the arcs $I_{j}$ and negative on the $\operatorname{arcs} I_{j}^{\prime}$.

We summarize this discussion by stating a theorem. Let us recall that we pick up one more parameter by allowing the pole of $f$ to vary freely, rather than being restricted to a fixed vertex. Also observe that the number $M$ of identified pairs of arcs cannot exceed half the number of zeroes (multiplicity counted) of $Q(z)$ which have modulus one. If $N_{1}$ is the number of zeroes of $Q$ of modulus less than one, the number of zeroes of $Q$ on the unit circle is just $2 N-2 N_{1}$.

THEOREM 6.1. Let

$$
f(z)=z+a_{2} z^{2}+\cdots+a_{N} z^{N}+a_{N+1} z^{N+1}+\ldots
$$

be an extremal schlicht function satisfying an equation

$$
\sum_{j=1}^{N} A_{j}(f(z))^{-j}\left(\frac{z f^{\prime}(z)}{f(z)}\right)^{2}=\sum_{j=-N}^{N} B_{j} z^{j}=Q(z),
$$

and let $N_{1}$ be the number of zeroes of $Q$ in the open unit disc.

Then $\partial \mathfrak{V}_{N+1}$ contains a $C^{\infty}$ submanifold through the point $\left(a_{2}, \ldots, a_{N+1}\right)$, and the real dimension of this submanifold is at least $\operatorname{Max}\left\{1,1+3 N_{1}-N\right\}$.

ACKNowledgement. A significant portion of the work leading to this paper was done at Glasgow University, 1970-1971, while the author was on sabbatical leave.

The author wishes to thank the referee for valuable comments and suggestions, and particularly for corrections to Theorem 4.1 and Lemma 5.1.

\section{REFERENCES}

1. R. Courant, Dirichlet's principle, conformal mapping, and minimal surfaces, Interscience, New York, 1950. MR 12, 90.

2. T. L. McCoy, Variation of conformal spheres by simultaneous sewing along several arcs, Trans. Amer. Math. Soc. 231 (1977), 65-82. 
3. A. C. Schaeffer and D. C. Spencer, Coefficient regions for schlicht functions, Amer. Math. Soc. Colloq. Publ., vol 35, Amer. Math. Soc., Providence, R. I., 1950. MR 12, 326.

4. M. Schiffer and D. C. Spencer, Functionals of finite Riemann surfaces, Princeton Univ. Press, Princeton, N. J., 1954. MR 16, 461.

Department of Mathematics, Michigan State Universtty, East Lansing, Michigan 48824

Current address: Department of Mathematics, University of New Mexico, Albuquerque, New Mexico 87106 